Research Paper

\title{
Clinicopathological features and relation between anaplastic lymphoma kinase (ALK) mutation and histological subtype of lung adenocarcinoma in Eastern European Caucasian population
}

Bojan Zaric ${ }^{1}$, Vladimir Stojsic ${ }^{1}$, Milana Panjkovic ${ }^{1}$, Dragana Tegeltija ${ }^{1}$, Vanesa Stepanov ${ }^{1}$, Tomi Kovacevic ${ }^{1}$,

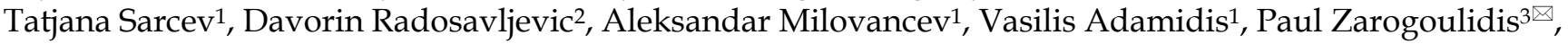
Wolfgang Hohenforst-Schmidt4, Georgia Trakada5 ${ }^{5}$ Aggeliki Rapti6, Branislav Perin ${ }^{1}$

1. Institute for Pulmonary Diseases of Vojvodina, Faculty of Medicine, University of Novi Sad, Serbia;

2. Institute of Oncology and Radiology of Serbia, Belgrade, Serbia;

Pulmonary Oncology Unit, "G. Papanikolaou” General Hospital, Aristotle University of Thessaloniki, Thessaloniki, Greece;

4. Medical Clinic I, "Fuerth" Hospital, University of Erlangen, Fuerth, Germany;

5. Division of Pulmonology, Department of Clinical Therapeutics, National and Kapodistrian University of Athens School of Medicine, Alexandra Hospital, Athens,

Greece;

6. Pulmonary Department, "Sotiria " Hospital of Chest Diseases, Athens, Greece.

$\triangle$ Corresponding author: Paul Zarogoulidis, M.D, Ph. D., Pulmonary Department-Oncology Unit, "G. Papanikolaou” General Hospital, Aristotle University of Thessaloniki, Thessaloniki, Greece. Fax: 00302310992424; Mobile: 00306977271974; E-mail: pzarog@hotmail.com.

(C) Ivyspring International Publisher. Reproduction is permitted for personal, noncommercial use, provided that the article is in whole, unmodified, and properly cited. See http://ivyspring.com/terms for terms and conditions.

Received: 2016.07.07; Accepted: 2016.09.04; Published: 2016.10.25

\begin{abstract}
Introduction: The incidence of echinoderm microtubule-associated protein-like4-anaplastic lymphoma kinase (EML4-ALK) mutation among surgically treated patients with adenocarcinoma of the lung of the Eastern European ethnicity is underreported. The aim of this trial was the determination of EML4-ALK mutation frequency in investigated population, and the evaluation of correlations between lung adenocarcinoma subtype and clinical characteristics with mutation status.

Patients and methods: This was a prospective trial which included 195 patients with adenocarcinoma of the lung who underwent surgical treatment. ALK mutation screening was performed by immunohistochemistry $(\mathrm{IHC})$. IHC scores of $2+$ and $3+$ were regarded as positive. Confirmatory FISH was performed in all IHC positive and in 2:1 ratio in negative patients.

Results: Overall ALK mutation rate established by IHC was $6.2 \%$, while FISH confirmed rate of $5.1 \%$. The FISH confirmed ALK positivity in 7.6\% Hungarians, 5.5\% Serbians, and $6.6 \%$ Slovakians. Acinar subtype of adenocarcinoma of the lung was significantly $(p=0.02)$ related to EML4-ALK positive mutation status. Most of the patients were males (56.9\%), smokers $(50.8 \%)$, or former smokers $(28.7 \%)$ with acinar $(55.4 \%)$ or solid (35.9\%) adenocarcinoma of the lung. Sensitivity and specificity of IHC were $100 \%$ and $98.9 \%$ respectively.

Conclusions: ALK mutation rate in surgically treated patients with adenocarcinoma of the lung was found to be $6.2 \%$ by IHC and $5.1 \%$ by FISH. Acinar subtype of the adenocarcinoma of the lung was significantly related to ALK positive mutation.
\end{abstract}

Key words: adenocarcinoma of the lung; anaplastic lymphoma kinase (ALK); EML4-ALK translocation; non-small cell lung cancer

\section{Introduction}

Lung cancer remains number one cause of cancer related morbidity and mortality worldwide. In past decade, we witnessed a significant revolution in understanding the molecular pathogenesis of lung cancer. There was a major shift in frequency of histology subtypes of non-small cell lung cancer 
(NSCLC); adenocarcinoma became major histological type alternating squamous cell lung cancer. The emergence of new oncogenic targets resulted in the development of new treatment options for lung cancer. The inhibitors for some of the most explored targets such as echinoderm growth factor receptor (EGFR), vascular endothelial growth factor receptor (VEGFR), and anaplastic lymphoma kinase (ALK) rearrangement are already in everyday therapeutic use. However, the frequency of these targets varies greatly in different populations. EGFR mutations are most common in the Far East Asian population, the frequency of VEGFR alteration is difficult to detect, and data on ALK mutation frequency are insufficient. ${ }^{1-3}$ This lack of data is one of the most significant obstacles for the introduction of novel therapies, especially in developing countries. Along with other factors (mainly financial) it usually prevents healthcare systems in adequate planning of healthcare budgets for oncological molecular (targeted) therapy. This results in the lack of reimbursement for such therapies in certain developing countries. ${ }^{3-7}$ The lack of data on Serbian population is one of the main reasons for reluctance of healthcare system to approve reimbursement of ALK inhibitors. Similar situation could be translated to the surrounding low income eastern European countries. Since the cost of molecular testing is also important issue, narrowing of indications, especially to the subtype of lung adenocarcinoma might be one of the justified strategies for decrease of molecular testing costs.

The prognostic and predictive values of ALK mutation in resected adenocarcinoma patients are proven in large scale trials, one of which originated from Europe ${ }^{6}$, however this trial did not evaluate the histology subtype of the adenocarcinoma. Since large differences exist in NSCLC common mutation rates among different races it is necessary to explore each region separately in order to establish true relationship and mutation frequency for specific geographic regions and populations.

The major aim of this trial was the determination of frequency in EML4-ALK mutation in patients with resected lung adenocarcinoma of the multiethnic population of Northern Serbian province of Vojvodina. Immunohistochemistry (IHC) screening was used for determination of ALK positive patients with lung adenocarcinoma while fluorescence in-situ hybridization (FISH) followed for final confirmation of results. Secondary objectives were: the evaluation of clinicopathological characteristics of patients in this population and the determination of relation between histology subtype of lung adenocarcinoma and ALK mutation status.

\section{Materials and Methods}

The study was a prospective, non-randomized trial, conducted at the Institute for Pulmonary Diseases of Vojvodina in the period from January 2012 to December 2014. The study was approved by the institutional review and ethics board. All of the patients who decided to participate in the study were informed about the genetic testing procedure, potential benefits and the risks, and all of them had signed institutional informed consent form.

Inclusion criteria were: age over 18 years; histological confirmation of lung adenocarcinoma after lung cancer surgery; chemotherapy, targeted therapy and radiotherapy naïve; and sufficient amount of tissue for genetic testing. Exclusion criteria were: non-adenocarcinoma of the lung histology/cytology, previous chemotherapy, radiotherapy or targeted therapy, and insufficient amount of tissue.

Patient demographic data as well as TNM status, stage and type of surgery and symptoms (cough, haemoptyses, dyspnea, hoarseness, loss of appetite, body weight loss, tumor associated chest pain, fever) were extracted from the institutional lung cancer registry and patient record database of the Institute for Pulmonary Diseases of Vojvodina.

All available pathology slides from each case were reviewed to verify the diagnosis and to select the representative block containing the most viable tumor cells by two pathologists. The IHC and FISH testing sequence is given in Figure 1. IHC scores of $2+$ and $3+$ were regarded as positive.

\section{Immunohistochemistry (IHC)}

Four-micron sections cut from formalin-fixed, paraffin-embedded blocks were placed on charged slides. Slides were deparaffinized and placed in working solution prepared by diluting Envison FLEX Target Retrieval Solution (50x) (DAKO, Glostrup, Denmark). Primary antibody (Mouse Monoclonal Anti-Human CD246, ALK protein, Clone ALK 1, Ready-to-use, DAKO, Glostrup, Denmark) was applied and incubated for 30 minutes. Visualization system EnVision FLEX+, Mouse, High pH (DAKO) with 15 minutes' incubation for En Vision FLEX+ Mouse (Link) and 20 minutes' incubation for En Vision FLEX+/HRP (Horseradishperoxidase) was performed. The positive control was from a known CD30-positive ALCL case. The negative control was a mouse immunoglobulin G1 serum substitution for the primary antibody (ALK).

An immunohistochemistry (IHC) score was assigned to each case according to the following criteria: 0, no staining (Figure 2.A); 1+, faint cytoplasmic staining in $\geq 10 \%$ of tumor cells (Figure 
2.B); 2+, moderate, smooth cytoplasmic staining (Figure 2.C and D); 3+, intense, granular cytoplasmic staining (Figure 2. D and F). IHC scoring was performed by two pathologists. Consensus was established by reviewing the slides together in the case of discrepant scores, typically $1+$ versus 0 .

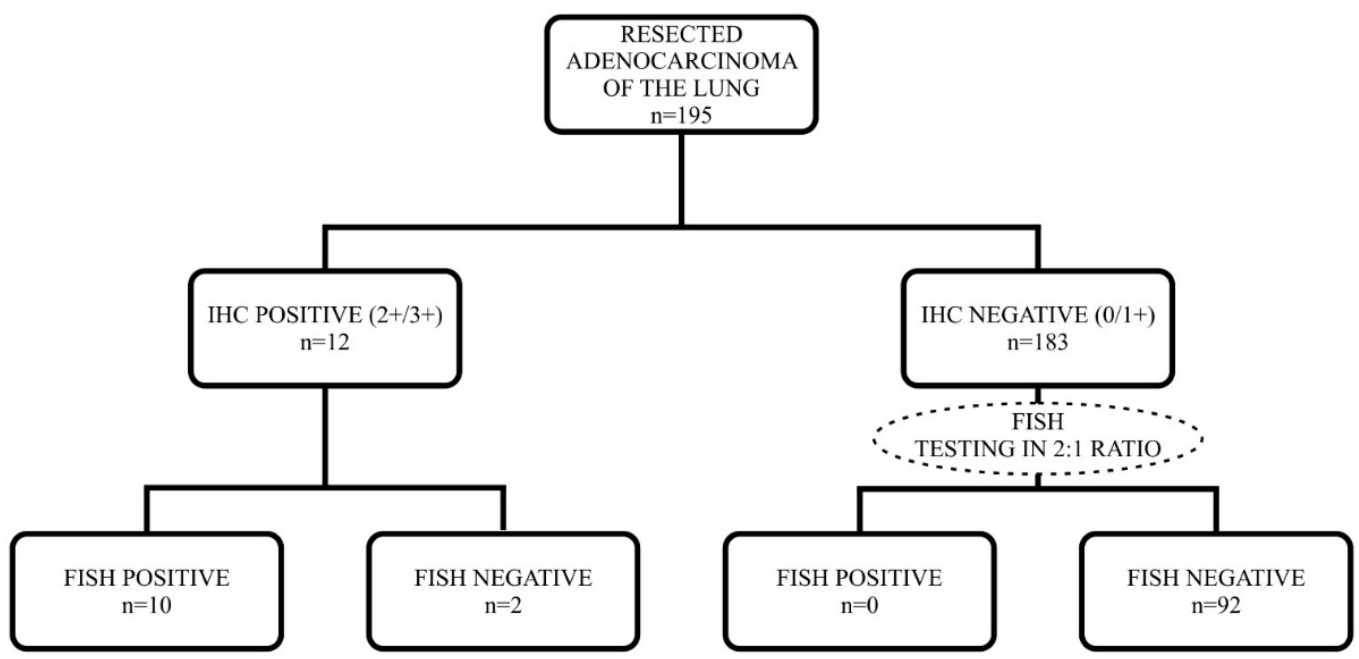

Figure 1. Flowchart of IHC and FISH testing in studied population.

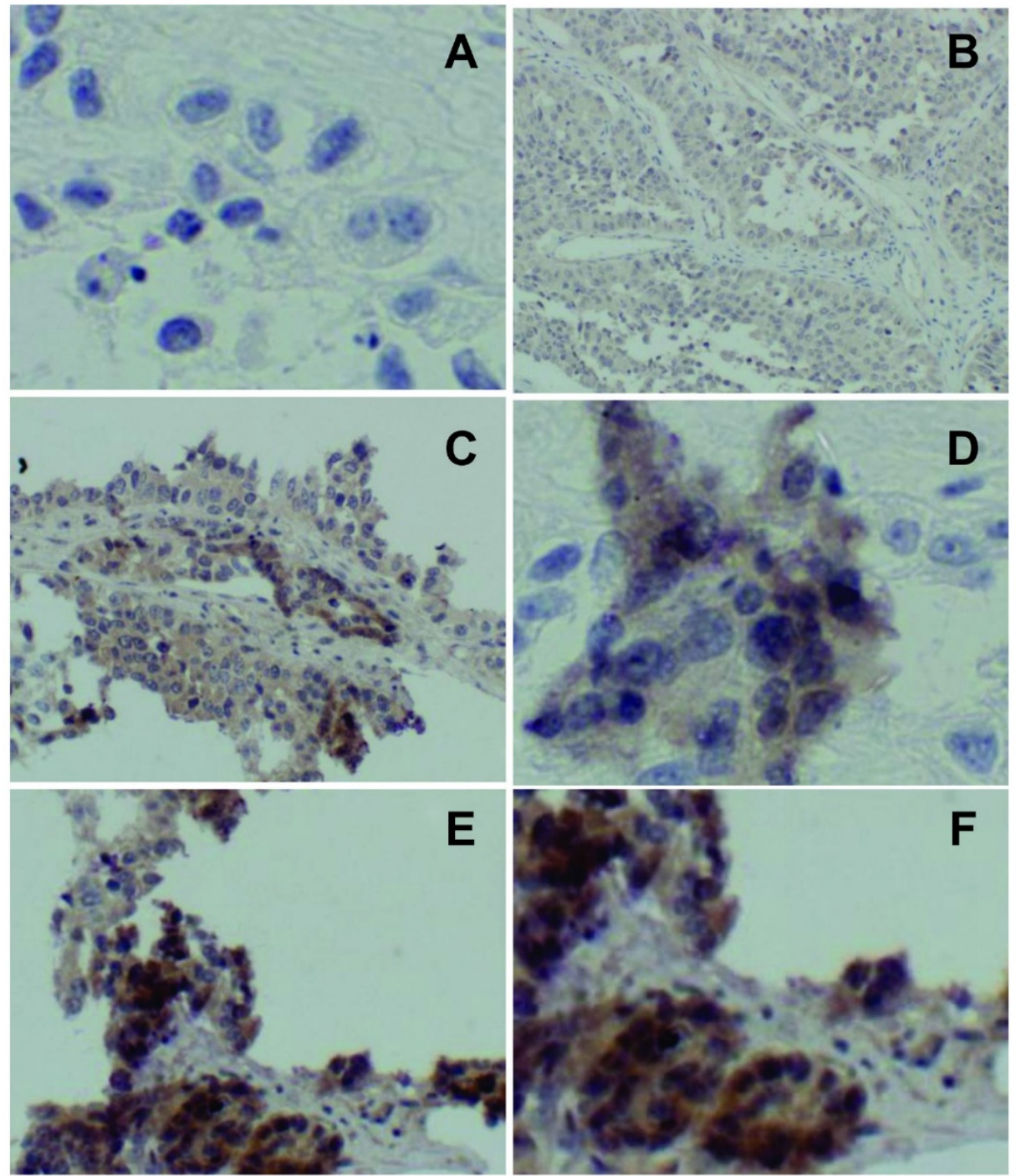

Figure 2. Score 0 showing no staining (A); score 1 showing faint, barely discernable cytoplasmic staining (B); score 2 showing moderate, smooth (without the apparent granularity seen in score 3) cytoplasmic staining (C, D); score 3 showing intense, granular cytoplasmic staining (E, F). 


\section{Fish}

FISH was performed in all IHC positive patients and in 2:1 ratio on IHC negative patients. Vysis ALK break-apart FISH probe was used according to instructions given in the package kit (Abbott Molecular, Des Plaines, IL, USA).

\section{Statistical analysis}

Descriptive statistics were generated for all study variables, including mean and standard deviation (SD) for continuous variables and relative frequencies for categorical variables. The $\chi^{2}$ test, the Fisher's exact test and the two-tailed Mann-Whitney test were used to assess the relation between mutation status and clinicopathological parameters. All probability values were calculated by assuming a 2-tailed a value of 0.05 with confidence intervals at the $95 \%$ level. All statistical analyses were performed with SPSS for Windows version 15.0 (SPSS Inc., Chicago, IL).

\section{Results}

There were 84 (43.1\%) female and 111 (56.9\%) male patients enrolled in this trial. Average age in the investigated population was $60.4 \pm 8.9$ years. Most of the patients, $148(75.9 \%)$ were Eastern Cooperative Oncology Group (ECOG) performance status 1, while ECOG 0 was found in $47(24.1 \%)$ patients. Majority of patients were active and former smokers; 99 (50.8\%) and $56(28.7 \%)$ respectively. There were only 40 (20.5\%) non-smokers. According to nationality, there were $126(64.6 \%)$ Serbian patients, 26 (13.3\%) Hungarian, 9 (4.6\%) Romanian, 15 (7.7\%) Slovakian, 9 (4.6\%) Roma, and 10 (5.1\%) Croatians. Most common subtypes of the adenocarcinoma of the lung were acinar, diagnosed in 108 (55.4\%) patients and solid detected in $70(35.9 \%)$ patients. The distribution of adenocarcinoma subtypes is given in table 1 . IHC testing revealed ELM4-ALK mutation in 12 (6.2\%) patients, while FISH testing confirmed 10 (5.1\%) positive results. The FISH testing confirmed ALK positivity in 2 (7.6\%) Hungarians, 7 (5.5\%) Serbians, and $1(6.6 \%)$ Slovakian patient. The data regarding TNM status, stage, and type of surgery are given in table 2. In a multivariate analysis, we found significant correlation between positive EML4-ALK mutation and acinar type of lung adenocarcinoma $(p=0.02)$. Association between smoking status and EML4-ALK mutation was marginally significant $(p=0.057)$. We did not find significant relations between demographic characteristics, TNM status, stage, investigated symptoms (cough, haemoptyses, dyspnea, hoarseness, loss of appetite, body weight loss, tumor associated chest pain, fever) and EML4-ALK mutation status. IHC testing showed sensitivity of $100 \%$ (95\% CI $69.97 \%-100 \%$ ), specificity 98.9\% (95\%CI $96.14 \%-99.84 \%)$, positive predictive value $83.33 \%$ (95\%CI $51.58 \%-97.42 \%)$ and negative predictive value $100 \%$ (95\% CI 97.98\%-100\%).

Table 1. Distribution of lung adenocarcinoma histology subtypes according to IASLC/ATS/ERS international multidisciplinary classification.

\begin{tabular}{lll}
\hline Adenocarcinoma subtype & Frequency & Percentage \\
\hline Lepidic & 5 & 2.6 \\
Acinar & 108 & 55.4 \\
Papillary & 10 & 5.1 \\
Micropapillary & 2 & 1.0 \\
Solid & 70 & 35.9 \\
Total & 195 & 100 \\
\hline IASLC = International Association for the Study of Lung Cancer, ATS = American
\end{tabular}

Thoracic Society, ERS = European Respiratory Society.

Table 2. TNM status, stage and type of resection in patients with resected adenocarcinoma of the lung.

\begin{tabular}{|c|c|c|}
\hline pT status & Frequency & Percent \\
\hline T1a & 20 & 10.3 \\
\hline $\mathrm{T} 1 \mathrm{~b}$ & 28 & 14.4 \\
\hline $\mathrm{T} 2 \mathrm{a}$ & 58 & 29.7 \\
\hline $\mathrm{T} 2 \mathrm{~b}$ & 31 & 15.9 \\
\hline $\mathrm{T} 3$ & 50 & 25.6 \\
\hline $\mathrm{T} 4$ & 8 & 4.1 \\
\hline Total & 195 & 100 \\
\hline \multicolumn{3}{|l|}{ pN status } \\
\hline No & 131 & 67.2 \\
\hline N1 & 42 & 21.5 \\
\hline N2 & 20 & 10.3 \\
\hline N3 & 2 & 1.0 \\
\hline Total & 195 & 100 \\
\hline \multicolumn{3}{|l|}{ M status } \\
\hline M0 & 190 & 97.4 \\
\hline M1a & 4 & 2.1 \\
\hline M1b & 1 & 0.5 \\
\hline Total & 195 & 100 \\
\hline \multicolumn{3}{|l|}{ Stage } \\
\hline IA & 28 & 14.4 \\
\hline IB & 39 & 20.0 \\
\hline IIA & 46 & 23.6 \\
\hline IIB & 43 & 22.1 \\
\hline IIIA & 32 & 16.4 \\
\hline IIIB & 3 & 1.5 \\
\hline IV & 4 & 2.1 \\
\hline Total & 195 & 100 \\
\hline \multicolumn{3}{|l|}{ Type of surgery } \\
\hline Pneumonectomy & 27 & 13.9 \\
\hline Lobectomy & 99 & 50.8 \\
\hline Segmentectomy & 9 & 4.6 \\
\hline Sleeve lobectomy & 36 & 18.4 \\
\hline Bilobectomy & 18 & 9.2 \\
\hline Exploratory Thoracotomy & 6 & 3.1 \\
\hline Total & 195 & 100 \\
\hline
\end{tabular}

\section{Discussion}

The overall EML4-ALK mutation frequency of $5.1 \%$ in the investigated population correlates with previously reported frequencies in other populations. The majority of reported results come from the Far 
East Asian trials and, unlike the EGFR, there is apparently no difference between the two major populations, Caucasian and Asian. However, there is obvious lack of data on frequency of ALK mutation in Eastern European populations. Certainly, one of the pitfalls of this trial is a relatively small number of positive patients. Significant correlation with acinar subtype of adenocarcinoma might be a result of the predominance of this subtype in the investigated population. One of the latest Chinese trials showed the same frequency of EML4-ALK mutation $(5.1 \%)$ as ours. The Patients in that trial had completely comparable clinicopathologic features to the ones observed in our trial. The investigators found higher prevalence of EML4-ALK mutation in invasive mucinous adenocarcinoma while we found it in acinar subtype. ${ }^{2}$ Comparable frequency of EML4-ALK mutation $(5.6 \%)$ and comparable populations were also confirmed in one of the most recent Chinese trials. ${ }^{3}$ In one of the largest European trials ${ }^{6}$, IHC revealed the same frequency in EML4-ALK mutation as in our trial $-6.2 \%$, but FISH confirmed significantly lower frequency (2.2\%). However, that trial did not evaluate the subtype of adenocarcinoma. On the other hand, the true determination of racial characteristics of population in large scale European trial might be difficult. Slightly higher frequency of EML4-ALK mutation $(10.7 \%$ by IHC and $9.5 \%$ by FISH) was reported in Korean population. Sensitivity and specificity of IHC (Novocastra, Clone 5A4, Newcastle, UK) screening was comparable to ours, $100 \%$ and $98.7 \%$, respectively. Two of the latest Korean trials 8,9 also reported higher frequency of EML4-ALK mutation than Chinese, European or our trial. In comparable but pre-selected non-smoker population, 9\% of patients were having EML4-ALK mutation confirmed by IHC and FISH tests. ${ }^{8,10}$ The Korean trial which also investigated the patients with resected adenocarcinoma of the lung ${ }^{9}$ confirmed EML4-ALK mutation in $8.6 \%$ of the cases. Slightly higher frequency in EML4-ALK mutation rate was reported in a Chinese trial from Hangzhou. The authors also regarded IHC score of $2+$ and $3+$ as IHC positive and applied FISH in these patients. Frequency of EML4-ALK mutation was found to be $8.4 \% .11$ Sensitivity and specificity of IHC in regard to FISH as a standard was comparable to ours, $100 \%$ and $97.9 \%$ respectively. The two most important meta-analyses on clinicopathological characteristics are published in 2014 by Chinese authors. ${ }^{12,13}$ Fan's meta-analysis revealed some important data in regard to race and histology.12 The overall frequency of EML4-ALK mutation was found to be $5.7 \%$ in unselected population and $7.2 \%$ in patients with adenocarcinoma of the lung. However, the frequency of EML4-ALK mutation ranged from $0.99 \%$ to $15 \%$ in unselected non-small cell lung cancer (NSCLC) groups. In the East Asian groups the frequency of EML4-ALK mutation was $4.77 \%$, in Caucasians $3.91 \%$, and in multi-ethnic groups $5.12 \%$. Some of the interracial differences were noted in this meta-analysis. The odds ratio of carrying EML4-ALK mutation was found to be reduced by $28 \%$ in males of Asian origin, while this was opposite in Caucasians. This meta-analysis confirmed that age, gender, race, smoking status, tumor stage and histology might be the potential sources of heterogeneity in clinical trials. In the last meta-analysis [13] pooled frequency of EML4-ALK mutation in adenocarcinoma of the lung was $6.85 \%$. This meta-analysis revealed that EML4-ALK mutation is highly correlated with never/light smoking history, female and adenocarcinoma histology. The EML4-ALK mutation frequency of $5.1 \%$ in our trial is comparable to other trials. The Absence of correlation with smoking status and gender might be a consequence of rather small number of positive patients.

\section{Conclusions}

The frequency of EML4-ALK mutation in the Eastern European population of Northern Serbia is $5.1 \%$ and stands in concordance with previously published results in other Caucasian populations. IHC with high sensitivity and solid specificity could be a reasonable option for screening the patients with adenocarcinoma of the lung. Its negative predictive value is of utmost importance to exclude false positive results. In our population EML4-ALK mutation is significantly related to acinar subtype of the lung adenocarcinoma.

\section{Acknowledgement}

The study was supported by the grant of the Serbian Ministry of Science and Technology, grant number 175056.

\section{Compliance with Ethical Standards}

The study has been performed in accordance with the ethical standards of the Declaration of Helsinki and its later amendments. The study was approved by the ethics committee of Institute for Pulmonary Diseases of Vojvodina. Written informed consent was obtained from each patient.

\section{Conflict of Interest}

The authors have no relevant affiliations or financial involvement with any organization or entity with a financial interest in or financial conflict with the subject matter or materials discussed in the manuscript. This includes employment, 
consultancies, honoraria, stock ownership or options, expert testimony, grants or patents received or pending, or royalties.

\section{References}

1. Korpanty GJ, Graham DM, Vincent MD, Leighl NB. Biomarkers That Currently Affect Clinical Practice in Lung Cancer: EGFR, ALK, MET, ROS-1, and KRAS. Frontiers in oncology. 2014; 4:204.

2. Pan Y, Zhang Y, Li Y, et al. ALK, ROS1 and RET fusions in 1139 lung adenocarcinomas: a comprehensive study of common and fusion pattern-specific clinicopathologic, histologic and cytologic features. Lung cancer. 2014;84(2):121-126.

3. $\mathrm{Hu} \mathrm{H}, \mathrm{Pan} \mathrm{Y}, \mathrm{Li}$ Y, et al. Oncogenic mutations are associated with histological subtypes but do not have an independent prognostic value in lung adenocarcinoma. OncoTargets and therapy. 2014; 7:1423-1437.

4. Peters S, Taron M, Bubendorf L, Blackhall F, Stahel R. Treatment and detection of ALK-rearranged NSCLC. Lung cancer. 2013;81(2):145-154.

5. Leighl NB, Rekhtman N, Biermann WA, et al. Molecular testing for selection of patients with lung cancer for epidermal growth factor receptor and anaplastic lymphoma kinase tyrosine kinase inhibitors: American Society of Clinical Oncology endorsement of the College of American Pathologists/International Association for the study of lung cancer/association for molecular pathology guideline. Journal of clinical oncology: official journal of the American Society of Clinical Oncology. 2014;32(32):3673-3679.

6. Blackhall FH, Peters S, Bubendorf L, et al. Prevalence and clinical outcomes for patients with ALK-positive resected stage I to III adenocarcinoma: results from the European Thoracic Oncology Platform Lungscape Project. Journal of clinical oncology: official journal of the American Society of Clinical Oncology. 2014;32(25):2780-2787.

7. Ye T, Pan Y, Wang R, et al. Analysis of the molecular and clinicopathologic features of surgically resected lung adenocarcinoma in patients under 40 years old. Journal of thoracic disease. 2014;6(10):1396-1402.

8. Sun JM, Lira M, Pandya K, et al. Clinical characteristics associated with ALK rearrangements in never-smokers with pulmonary adenocarcinoma. Lung cancer. 2014;83(2):259-264.

9. Kim MH, Shim HS, Kang DR, et al. Clinical and prognostic implications of ALK and ROS1 rearrangements in never-smokers with surgically resected lung adenocarcinoma. Lung cancer. 2014;83(3):389-395.

10. Park HS, Lee JK, Kim DW, et al. Immunohistochemical screening for anaplastic lymphoma kinase (ALK) rearrangement in advanced non-small cell lung cancer patients. Lung cancer. 2012;77(2):288-292.

11. Zhou J, Zhao J, Sun K, et al. Accurate and economical detection of ALK positive lung adenocarcinoma with semiquantitative immunohistochemical screening. PloS one. 2014;9(3): e92828.

12. Fan L, Feng Y, Wan H, Shi G, Niu W. Clinicopathological and demographical characteristics of non-small cell lung cancer patients with ALK rearrangements: a systematic review and meta-analysis. PloS one. 2014;9(6): e100866.

13. Wang Y, Wang S, Xu S, Qu J, Liu B. Clinicopathologic features of patients with non-small cell lung cancer harboring the EML4-ALK fusion gene: a meta-analysis. PloS one. 2014;9(10): e110617. 